\title{
LOCALIZATION, HOMOLOGY AND A CONSTRUCTION OF ADAMS
}

\author{
BY \\ ARISTIDE DELEANU AND PETER HILTON (1)
}

\begin{abstract}
In recent papers, the authors have developed the technique of using Kan extensions to obtain extensions of homology and cohomology theories from smaller to larger categories of topological spaces. In the present paper, it is shown that the conditions imposed there to guarantee that the Kan extension of a cohomology theory is again a cohomology theory in fact also imply that the Kan extension commutes with stabilization. A construction, due to Adams, for completing a space with respect to a homology theory by using categories of fractions is generalized to triangulated categories, and it is shown that, for any family of primes $P$, the Adams completion of a space $X$ with respect to the homology theory $\tilde{H}_{*}\left(-; \mathbf{Z}_{P}\right)$ is the localization of $X$ at $P$ in the sense of Sullivan. Using this, the Kan extension of the restriction of a homology theory to the category of spaces having $P$-torsion homotopy groups is determined.
\end{abstract}

1. Introduction. We bring together in this paper three ideas drawn from current work in algebraic topology. The first ingredient is the localization theory of Sullivan [12], Kan-Bousfield [2], and others; the second is the technique of using Kan extensions to obtain (Čech) extensions of cohomology theories from smaller to larger categories of topological spaces, developed by the authors ([3],[5]); and the third is a construction, due to Adams, for 'representing' homology theories in the appropriate category of fractions, or, as Adams expressed it, completing a space with respect to a homology theory (see Theorem 3.2 for details).

One of our principal observations, which appears as an application of Theorem 3.2, is that Adams' construction is a generalization of localization. This becomes completely obvious if one remarks that there is another universal property, different from that given by Sullivan in [12], which characterizes the localization. Let $P$ be a family of primes. Then if $X$ is 1 -connected (or if we work in some suitable stable category) the $P$-localization of $X$ is a space $X_{P}$, together with a homotopy class of maps $e: X \rightarrow X_{P}$, such that (i) the homology of $X_{P}$ is $P$-local, (ii) the homology homorphism induced by $e P$-localizes the (reduced) integral homology $\tilde{H}_{*}(X)$, and (iii) if $f: X \rightarrow Y$ is a homotopy class and $\tilde{H}_{*}(Y)$ is $P$-local, there is a unique homotopy class $u: X_{P} \rightarrow Y$ with $u e=f$. In this characterization (where, in fact, as shown in [12], (ii) is a consequence of (i) and (iii)), we note that $\tilde{H}_{*}\left(X_{P}\right)$ is $P$-local and that $e$ sets up an isomorphism in homology with $\mathbf{Z}_{P}$ coefficients, where $\mathbf{Z}_{P}$ is the integers localized at $P$; and then ask that $X_{P}$ be

Presented in part to the Society, January 25, 1973 under the title Localization in homotopy theory and a construction of Adams; received by the editors May 28, 1971 and, in revised form, March 2, 1972.

AMS (MOS) subject classifications (1970). Primary 55B20; Secondary 55B05, 55B10, 18 A40.

Key words and phrases. Localization, homology, Kan extension, completion, Serre class, stabilization.

(1) Research partially supported by National Science Foundation grant GP-29107. 
universal with respect to the first property. Instead we may ask that $X_{P}$ be couniversal with respect to the second property.

Theorem 1.1. The localization $e: X \rightarrow X_{P}$ has property (iii'): given any homotopy class $f: X \rightarrow Y$ which induces an isomorphism in homology with $\mathbf{Z}_{P}$ coefficients, there exists a unique homotopy class $v: Y \rightarrow X_{P}$ with $v f=e$.

Before proving this we remark that, since (ii) and (iii') determine $e$ up to canonical equivalence, it follows that they do characterize the $P$-localization. To prove the theorem we invoke a lemma.

Let $C_{P}$ be the Serre class of $P$-torsion groups, and let $\bar{P}$ be the family of primes complementary to $P$.

Lemma 1.2. If $H \in C_{\bar{P}}$, then $\operatorname{Hom}(H, G)=0, \operatorname{Ext}(H, G)=0$ for any $\mathbf{Z}_{P}$-module G.

Proof. Write $n \in \bar{P}$ if $n$ is a product of primes in $\bar{P}$. Let $\phi: H \rightarrow G$ and $a \in H$. Then $n a=0, n \in \bar{P}$, so $n \phi(a)=0$. But $1 / n \in \mathbf{Z}_{P}$, so $\phi(a)=0, \phi=0$.

Now let $G \mapsto I \rightarrow J$ be an injective presentation of $G$ as a $\mathbf{Z}_{P}$-module. Then $I$ is certainly divisible as an abelian group, so that $\operatorname{Ext}(H, G)$ is a quotient of $\operatorname{Hom}(H, J)$ and hence zero.

Proof of Theorem 1.1. The obstructions to existence and uniqueness of $v$ lie in $H^{*}\left(f ; \pi_{*}\left(X_{P}\right)\right)$. Now $H_{*}(f) \otimes \mathbf{Z}_{P}=0$, since $f$ induces a homology isomorphism with $\mathbf{Z}_{P}$ coefficients. It follows that $H_{*}(f) \in C_{\bar{P}}$ (Lemma 4.3), so that the universal coefficient theorem in cohomology, together with Lemma 1.2, yields a proof of the theorem.

This characterization of localization provides, as we say, an immediate link between localization and the Adams construction. In fact, we show, using Theorem 3.2, which is a generalization to triangulated categories of a result of Adams, that the $P$-localization is effectively just the Adams completion with respect to the homology theory $\tilde{H}_{*}\left(-; \mathbf{Z}_{P}\right)$. Furthermore, we note that the $p-$ profinite completion in the sense of Sullivan [12] is just the Adams completion with respect to the homology theory $\tilde{H}_{*}(-; \mathbf{Z} / p \mathbf{Z})$.

The link between the Adams construction and the Kan extension of a homology theory was suggested by the notion to consider the (singular) Kan extension of homology instead of the (Čech) Kan extension of cohomology. This is in many ways preferable, but it seems that, for homology, one is constrained to work stably. We work in the stable category of CW-complexes, since excellent accounts of this category occur in the literature. However, we might have chosen to work in the Boardman category of spectra, and we do in fact indicate in the text, as an application of Theorem 3.2, that the Adams completion always exists in the Boardman category. It turned out (Theorem 2.2) that the conditions we imposed in [5] to guarantee that the Kan extension of a cohomology theory is again a cohomology theory in fact also imply that the Kan extension commutes with stabilization.

Now the following theorem was proved in [5]. 
Theorem 1.3 (Theorem 4.1 of [5]). Let $h$ be a representable cohomology theory with coefficients of finite type and let $h_{0}$ be its restriction to the category of 1connected finite $C W$-complexes belonging to $C_{P}$. Then the Kan extension $h_{1}$ of $h_{0}$ to the category of 1-connected finite-dimensional $C W$-complexes is given by $h_{1}^{n}(X)$ $=h^{n-1}\left(X ; \mathbf{Z}_{P^{\infty}}\right)$.

Here we say that $Y$ belongs to $C_{P}$ if $\tilde{H}_{*}(Y) \in C_{P}$; and $\mathbf{Z}_{P^{\infty}}$ is the generalized Prüfer group, inserted as a coefficient group into $h$ as in [10]. In this paper we prove a homology analog of this theorem (Theorem 4.4), but our approach is quite different. We note that $Y \in C_{P}$ if and only if $\tilde{H}_{*}\left(Y ; \mathbf{Z}_{\bar{P}}\right)=0$, where $\bar{P}$ is the set of primes complementary to $P$. If $e: X \rightarrow X_{\bar{P}}$ is the $\bar{P}$-localization of $X$ (or, equivalently, the Adams completion with respect to $\tilde{H}_{*}\left(-; \mathbf{Z}_{\bar{P}}\right)$ ), then $C_{e}$, the mapping cone of $e$, belongs to $C_{P}$, and we show that, working stably, the desuspension of $C_{e}$ is cofinal among the stable objects over $X$ which belong to $C_{P}$.

In establishing that Kan extension commutes with stabilization, and again in proving Theorem 4.4, we make essential use of the notion of cofinality of functors on filtering categories [1]; we have given a treatment, adapted to our needs, in [4]. However, to facilitate the reading of this paper, let us briefly recall here the relevant facts. We say that the category $\mathbf{I}$ is filtering if

$$
\text { given } i_{1}, i_{2} \text { in } \mathbf{I} \text {, there exists }{ }_{i_{2}}^{i_{1}} \rightarrow \text { in } \mathbf{I} \text {; }
$$

$$
\text { given } i \underset{\phi^{\prime}}{\stackrel{\phi}{\rightleftarrows}} i^{\prime} \text { in I, there exists } \psi \text { with } \psi \phi=\psi \phi^{\prime} \text {. }
$$

We say that the functor $T: \mathbf{I}_{0} \rightarrow \mathbf{I}$ is cofinal, if

$$
\text { given } i \text { in } \mathbf{I} \text {, there exists } i \rightarrow T\left(i_{0}\right) \text { in } \mathbf{I} \text { with } i_{0} \text { in } \mathbf{I}_{0} \text {; }
$$

(ii) given $i \underset{\psi_{2}}{\stackrel{\psi_{1}}{\longrightarrow} T\left(i_{1}\right)}$ in $\mathbf{I}$, there exists $\underset{i_{2}}{i_{1} \underset{\phi_{2}}{\phi_{1}} j} j$ in $\mathbf{I}_{0}$ with $T\left(\phi_{1}\right) \psi_{1}=T\left(\phi_{2}\right) \psi_{2}$.

As a special case of Theorem 2.10 of [4] we have

Theorem 1.4. Let $\mathbf{I}_{0} \stackrel{T}{\rightarrow} \mathbf{I} \stackrel{F}{\rightarrow}$ C be functors with $T$ cofinal, and assume that the category $\mathbf{I}_{0}$ is filtering and the category $\mathbf{C}$ is cocomplete. Then

$$
\lim _{\rightarrow} F T=\lim _{\rightarrow} F
$$

The reader should note that we are often concerned in this paper with contravariant functors, so that we may have to look at properties of the categories $\mathbf{I}_{0}$ and $\mathbf{I}$ and the functor $T$ dual to those described above.

The authors are very grateful to the referee for suggesting certain improvements to an earlier draft of this paper.

2. Stabilization and Kan extension. Let $\mathbf{T}$ be a triangulated category with suspension automorphism $\Sigma$ [8]. Then a cohomology functor $h: \mathbf{T} \rightarrow \mathbf{A}$, where $\mathbf{A}$ is an abelian category, is a contravariant functor such that, for any triangle 


$$
X \stackrel{u}{\longrightarrow} Y \stackrel{v}{\longrightarrow} Z \stackrel{w}{\longrightarrow} \Sigma X
$$

in $\mathbf{T}$, the sequence

$$
\begin{aligned}
\ldots \rightarrow & h\left(\Sigma^{i+1} X\right) \stackrel{h\left(\Sigma^{i} w\right)}{\longrightarrow} h\left(\Sigma^{i} Z\right) \stackrel{h\left(\Sigma^{i} v\right)}{\longrightarrow} h\left(\Sigma^{i} Y\right) \stackrel{h\left(\Sigma^{i} u\right)}{\longrightarrow} h\left(\Sigma^{i} X\right) \\
& \rightarrow \ldots
\end{aligned}
$$

in $\mathbf{A}$ is exact.

Now let $\mathbf{T}_{1}$ be a triangulated category, $\mathbf{T}_{0}$ a full triangulated subcategory of $\mathbf{T}_{1}$, and $h: \mathbf{T}_{0} \rightarrow \mathbf{A b}$ a cohomology functor to abelian groups. We may construct the Kan extension $h_{1}: \mathbf{T}_{1} \rightarrow \mathbf{A b}$ of $h$. We may then prove

Theorem 2.1. If $\mathbf{T}_{0}$ has weak local pullbacks rel $\mathbf{T}_{1}$, then $h_{1}$ is a cohomology functor.

Proof. The argument closely resembles that of Theorem 2.14 of [5]. It is sufficient to show that, given (2.1) in $\mathbf{T}_{1}$, then

$$
h_{1}(Z) \stackrel{h_{1}(v)}{\longrightarrow} h_{1}(Y) \stackrel{h_{1}(u)}{\longrightarrow} h_{1}(X)
$$

is exact. Since $\mathbf{T}_{0}$ admits finite products and weak local pullbacks rel $\mathbf{T}_{1}$, it follows that, for any $Y$ in $\mathbf{T}_{1}$, an element $\xi \in h_{1}(Y)$ is represented by a pair $(\alpha, f)$ where $f: Y \rightarrow V, V$ in $\mathbf{T}_{0}$, and $\alpha \in h(V)$. Moreover, $(\alpha, f)$ represents 0 if and only if we have $f=d g$,

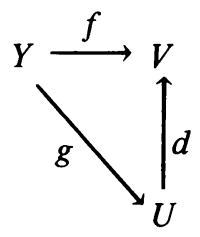

with $d$ in $\mathbf{T}_{0}$ and $h(d) \alpha=0$. We write $\xi=[\alpha, f]$, so that $h_{1}(u) \xi=[\alpha, f u]$. Thus if $h_{1}(u) \xi=0$, then we have a diagram

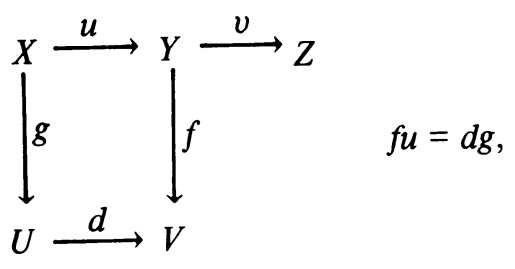

with $h(d) \alpha=0$. Invoking the axioms for a triangulated category and the fact that $h$ is a cohomology functor, we may complete $(2.3)$ to the commutative diagram 


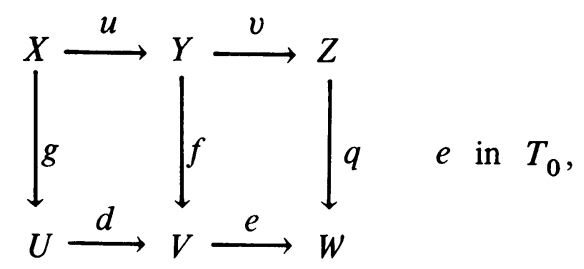

and find $\beta \in h(W)$ with $h(e) \beta=\alpha$. Then

$$
h_{1}(v)[\beta, q]=[\beta, q v]=[\beta, e f]=[\alpha, f]=\xi .
$$

This proves the theorem. Now the condition that $\mathbf{T}_{0}$ has finite products and weak local pullbacks rel $\mathbf{T}_{1}$ is just the condition that $\mathbf{T}_{0}$ is $\mathbf{T}_{1}$-adapted in the language of [5]. Thus, if we work stably, only the first condition imposed in [5] on the pair of categories in question is required to guarantee that the Kan extension of a cohomology functor is again a cohomology functor. We will now show that the second condition imposed in [5] permits us to work stably. This second condition is that of local right adjunctability of the suspension functor, as explained below.

Suppose given a pair of categories $\mathbf{J}_{1}, \mathbf{J}_{0}$ suitable for cohomology and assume $\mathbf{J}_{0}$ is $\mathbf{J}_{1}$-adapted. Then we saw in [5] that if, in addition, $\boldsymbol{\Sigma}$ is locally right $\tilde{\mathbf{J}}_{0}$ adjunctable, where $\tilde{\mathbf{J}}_{0}$ is the homotopy category of $\mathbf{J}_{0}$, then the Kan extension $h_{1}$ to $\mathbf{J}_{1}$ of a cohomology theory $h$ on $\mathbf{J}_{0}$ to $\mathbf{A b}$ is again a cohomology theory. Let us, for convenience, recall from [5] the definition of local right adjunctability, which may be stated for any functor $F: \mathbf{C}_{1}, \mathbf{C}_{0} \rightarrow \mathbf{D}_{1}, \mathbf{D}_{\mathbf{0}}$ of one pair of categories to another. Then $F$ is locally right $\mathbf{C}_{0}$-adjunctable if

(i) given $\phi: F X \rightarrow Y$ in $\mathbf{D}_{1}, X$ in $\mathbf{C}_{1}, Y$ in $\mathbf{D}_{0}$, there exist $\psi: X \rightarrow Z$ in $\mathbf{C}_{1}, Z$ in $\mathbf{C}_{0}$, and $\theta: F Z \rightarrow Y$ in $\mathbf{D}_{0}$ with $\theta \circ F \psi=\phi$;

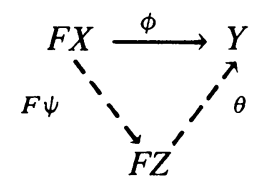

(ii) given $\theta_{1} \circ F \psi_{1}=\theta_{2} \circ F \psi_{2}$ as in (i), there exist $\beta_{i}: Z_{i} \rightarrow Z$ in $\mathbf{C}_{0}, i=1,2$, $\gamma: F Z \rightarrow Y$ in $D_{0}$, such that $\beta_{1} \psi_{1}=\beta_{2} \psi_{2}, \gamma \circ F \beta_{i}=\theta_{i}, i=1,2$.
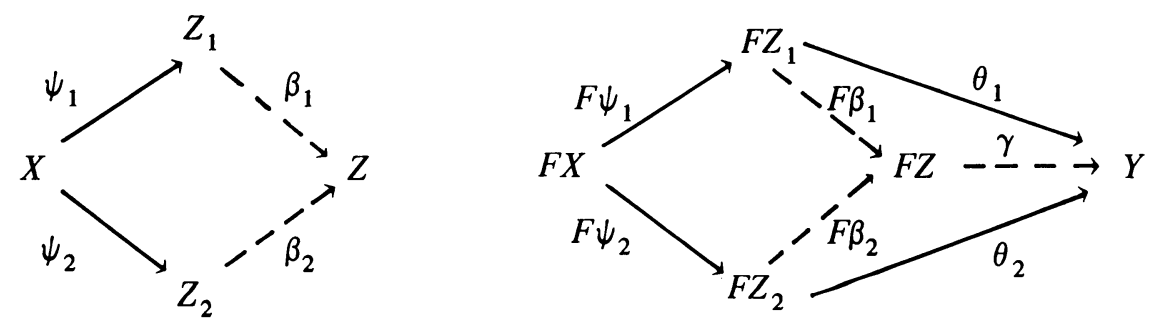

In this paper we are only concerned with the case where $\left(\mathbf{C}_{1}, \mathbf{C}_{0}\right)=\left(\mathbf{D}_{1}, \mathbf{D}_{0}\right)$; however, some lemmas we prove below have obvious generalizations.

Now let $\mathbf{J}_{\mathbf{0}}$ be $\mathbf{J}_{1}$-adapted, and let $\mathbf{S}_{1}, \mathbf{S}_{\mathbf{0}}$ be the stabilizations [9] of $\mathbf{J}_{1}, \mathbf{J}_{\mathbf{0}}$. The cohomology theory $h$ on $\mathbf{J}_{0}$ yields a cohomology functor $h^{s}: \mathbf{S}_{0} \rightarrow$ Ab by the rule 


$$
h^{s}(L, m)=h^{-m}(L), \quad L \text { in } \mathbf{J}_{0} .
$$

We call $h^{s}$ the stabilization of $h$. Similarly, $h_{1}$ yields the stabilization $h_{1}^{s}: \mathbf{S}_{1}$ $\rightarrow \mathbf{A b}$, where

$$
h_{1}^{s}(K, m)=h_{1}^{-m}(K), \quad K \text { in } \mathbf{J}_{1} .
$$

Now it is easy to see that if $\mathbf{J}_{0}$ is $\mathbf{J}_{1}$-adapted, then the pair $\left(\mathbf{S}_{1}, \mathbf{S}_{0}\right)$ satisfies the hypothesis of Theorem 2.1 for $\left(\mathbf{T}_{1}, \mathbf{T}_{0}\right)$. Thus the Kan extension $\left(h^{s}\right)_{1}: \mathbf{S}_{1} \rightarrow \mathbf{A b}$ is a cohomology functor. However, we now prove

Theorem 2.2. If $\Sigma$ is locally right $\tilde{\mathbf{J}}_{0}$-adjunctable, then

$$
h_{1}^{s}=\left(h^{s}\right)_{1}: \mathbf{S}_{1} \rightarrow \mathbf{A b} \text {. }
$$

In other words, stabilization commutes with Kan extension.

Proof. We proceed by establishing a sequence of lemmas.

Lemma 2.3. If the functors $F, G: \tilde{\mathbf{J}}_{1}, \tilde{\mathbf{J}}_{0} \rightarrow \tilde{\mathbf{J}}_{1}, \tilde{\mathbf{J}}_{0}$ are locally right $\tilde{\mathbf{J}}_{0}$-adjunctable, so is $G F$.

Proof. (i) Suppose given $f: G F X \rightarrow Y$ in $\tilde{\mathbf{J}}_{1}$ with $Y$ in $\tilde{\mathbf{J}}_{0}$. Since $G$ is locally right adjunctable, there exist $g_{1}: F X \rightarrow Z_{1}$ in $\tilde{\mathbf{J}}_{1}, u_{1}: G Z_{1} \rightarrow Y$ in $\tilde{\mathbf{J}}_{0}$ with $u_{1} \circ G g_{1}=f$. Since $F$ is locally right adjunctable, there exist $g: X \rightarrow Z$ in $\tilde{\mathbf{J}}_{1}$ $u_{2}: F Z \rightarrow Z_{1}$ in $\tilde{\mathbf{J}}_{0}$ with $g_{1}=u_{2} \circ F g$. Then $f=u \circ G F g$, with $u=u_{1} \circ G u_{2}$.

(ii) Suppose given $g_{i}: X \rightarrow Z_{i}$ in $\tilde{\mathbf{J}}_{1}, u_{i}: G F Z_{i} \rightarrow Y$ in $\tilde{\mathbf{J}}_{0}, i=1,2$, with $u_{1} \circ G F g_{1}=u_{2} \circ G F g_{2}$. Since $G$ is locally right adjunctable, we may find $k_{i}: F Z_{i} \rightarrow Z_{0}$ and $w_{0}: G Z_{0} \rightarrow Y$ in $\tilde{\mathbf{J}}_{0}, i=1,2$, such that $k_{1} \circ F g_{1}=k_{2} \circ F g_{2}$ and $w_{0} \circ G k_{i}=u_{i}, i=1,2$. Since $F$ is locally right adjunctable, we may find $v_{i}: Z_{i} \rightarrow Z$ and $l: F Z \rightarrow Z_{0}$ in $\tilde{\mathbf{J}}_{0}$, such that $v_{1} g_{1}=v_{2} g_{2}$ and $l \circ F v_{i}=k_{i}$, $i=1$, 2. But then, if $w=w_{0} \circ G l: G F Z \rightarrow Y$, then $w \circ G F v_{i}=u_{i}, i=1,2$.

Corollary 2.4. If $\Sigma$ is locally right $\tilde{\mathbf{J}}_{0}$-adjunctable, so is $\Sigma^{n}, n \geq 1$.

Lemma 2.5. If $F: \tilde{\mathbf{J}}_{1}, \tilde{\mathbf{J}}_{0} \rightarrow \tilde{\mathbf{J}}_{1}, \tilde{\mathbf{J}}_{0}$ is locally right $\tilde{\mathbf{J}}_{0}$-adjunctable then, given $g_{i}: X \rightarrow Z_{i}$ in $\tilde{\mathbf{J}}_{1}$, with $Z_{i}$ in $\tilde{\mathbf{J}}_{0}$, and $u_{i}: F Z_{i} \rightarrow Y$ in $\tilde{\mathbf{J}}_{0}, i=1$, , with $u_{1} \circ F g_{1}$ $=u_{2} \circ F g_{2}$, there exist $g_{0}: X \rightarrow Z_{0}, w_{i}: Z_{0} \rightarrow Z_{i}$ in $\tilde{\mathbf{J}}_{0}, i=1,2$, with $w_{i} \circ g_{0}=g_{i}$, $i=1,2$, and $u_{1} \circ F w_{1}=u_{2} \circ F w_{2}$.
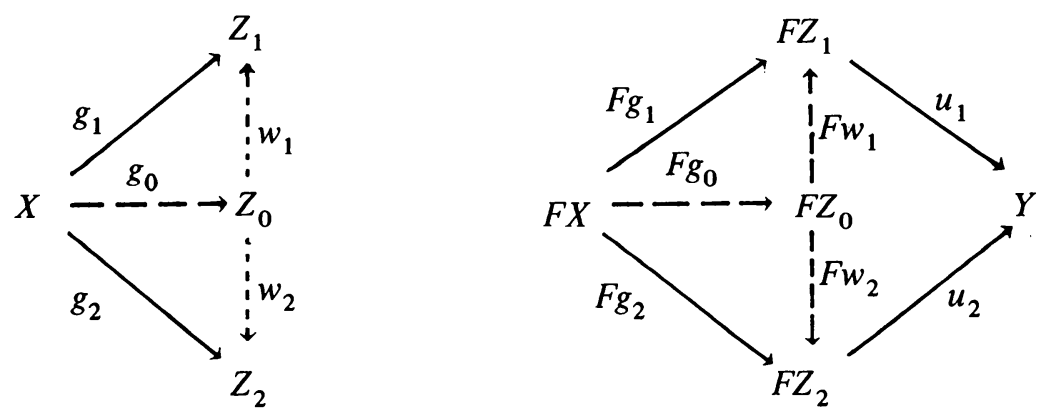
Proof. Since $F$ is locally right adjunctable, there exist $v_{i}: Z_{i} \rightarrow Z$ in $\tilde{\mathbf{J}}_{0}$, $w: F Z \rightarrow Y$ in $\tilde{\mathbf{J}}_{0}$, with $v_{1} g_{1}=v_{2} g_{2}, w \circ F v_{i}=u_{i}, i=1,2$. Since $\mathbf{J}_{0}$ is $\mathbf{J}_{1}$ adapted, there exist $g_{0}: X \rightarrow Z_{0}, w_{i}: Z_{0} \rightarrow Z_{i}$ in $\tilde{\mathbf{J}}_{0}, i=1,2$, with $w_{i} \circ g_{0}=g_{i}$, $i=1,2$, and $v_{1} w_{1}=v_{2} w_{2}$. Then $u_{1} \circ F w_{1}=w \circ F v_{1} \circ F w_{1}=w \circ F v_{2} \circ F w_{2}=u_{2}$ ○ $F w_{2}$.

Now, given the pair of categories $\mathbf{J}_{1}, \mathbf{J}_{0}$, let $\mathbf{S}_{1}$ and $\mathbf{S}_{0}$ be the stabilizations of $\mathbf{J}_{1}$ and $\mathbf{J}_{0}$, and let $(X, m)$ be an arbitrary object of $\mathbf{S}_{1}$. Consider the following two categories. $\mathbf{I}_{0}$ is the category $\tilde{\mathbf{J}}_{10}(X)$ of $\tilde{\mathbf{J}}_{0}$-objects under $X$ as defined in [5], and $\mathbf{I}_{1}$ is the category of $\mathbf{S}_{0}$-objects under $(X, m)$. As in [5, Theorem 2.12] it will follow that $\mathbf{I}_{0}$ is adapted; in particular, $\mathbf{I}_{0}^{\text {opp }}$ is filtering.

Let $Q: \mathbf{I}_{0} \rightarrow \mathbf{I}_{1}$ be the functor which sends the object $f: X \rightarrow Y$ of $\mathbf{I}_{0}$ into the object $\phi:(X, m) \rightarrow(Y, m)$ of $\mathbf{I}_{1}$, where the morphism $\phi$ in $\mathbf{S}_{1}$ is represented by $\Sigma^{m+k} f: \Sigma^{m+k} X \rightarrow \Sigma^{m+k} Y$ for any $k \geq-m$.

Lemma 2.6. If $\Sigma$ is locally right $\tilde{\mathbf{J}}_{0}$-adjunctable, then $Q: \mathbf{I}_{0}^{\text {opp }} \rightarrow I_{1}^{\text {opp }}$ is cofinal.

Proof. The first cofinality condition is an immediate consequence of the first condition that $\Sigma^{m}$ is locally right adjunctable. The second cofinality condition is an immediate consequence of Lemma 2.5 , applied to some suitably high iterate $\Sigma^{N}$ of $\Sigma$.

We are now ready to complete the proof of Theorem 2.2. Consider the object $(X, m)$ in $\mathbf{S}_{1}$. Then

$$
h_{1}^{s}(X, m)=h_{1}^{-m}(X)=\lim _{\overrightarrow{\mathbf{I}_{0}}} F_{0},
$$

where $F_{0}: \mathbf{I}_{0} \rightarrow \mathbf{A b}$ is the contravariant functor given by $F_{0}(g)=h^{-m}(Z)$ for $g: X \rightarrow Z$ in $\mathbf{I}_{0}$. On the other hand,

$$
\left(h^{s}\right)_{1}(X, m)=\lim _{\overrightarrow{\mathbf{I}_{1}}} F_{1}
$$

where $F_{1}: \mathbf{I}_{1} \rightarrow \mathbf{A b}$ is the contravariant functor given by $F_{1}(f)=h^{s}(Y, n)$ $=h^{-n}(Y)$ for $f:(X, m) \rightarrow(Y, n)$ in $\mathbf{I}_{1}$. Plainly $F_{1} Q=F_{0}$, so Lemma 2.6 and Theorem 1.4 show that

$$
\lim _{\overrightarrow{\mathbf{I}_{0}}} F_{0}=\lim _{\overrightarrow{\mathbf{I}_{1}}} F_{1}
$$

This completes the proof of Theorem 2.2.

Remark. Suppose $\mathbf{J}_{0}$ is $\mathbf{J}_{1}$-adapted, that $\Sigma$ satisfies the first condition for local right $\mathbf{J}_{0}$-adjunctability, and that $\Sigma$ satisfies the conclusion of Lemma 2.5 for $F=\Sigma$. Then it is easy to see that the Kan extension $\sigma_{1}$ of the suspension equivalence $\sigma: h^{n} \rightarrow h^{n+1} \Sigma$ is an equivalence for any cohomology theory $h$ on $\mathbf{J}_{0}$, so that the Kan extension $h_{1}$ of $h$ is a cohomology theory (compare Theorem 3.5 of [5]). On the other hand it is not clear that if $\Sigma$ has the properties assumed at the outset of this remark, then $\Sigma^{n}$ also has these properties, so that, although this weaker hypothesis on $\Sigma$ would have served in [5], it may well not be true that it would have implied Theorem 2.2. 
3. The Adams construction. Theorem 2.2 effectively permits us to work stably if we wish to study the Kan extension of a cohomology theory. We turn now to homology and continue to work stably. In fact we consider a homology functor $h: \mathbf{T}_{0} \rightarrow \mathbf{A b}$ defined on a triangulated category $\mathbf{T}_{0}$, and consider the question of extending $h$ via the Kan extension, to a functor $h^{1}: \mathbf{T}_{1} \rightarrow \mathbf{A b}$ on a triangulated category $\mathbf{T}_{1}$ containing $\mathbf{T}_{0}$ as a full subcategory. We have the immediate analog of Theorem 2.1, namely,

Theorem 3.1. If $\mathbf{T}_{0}$ has weak local pushouts rel $\mathbf{T}_{1}$, then $h^{1}$ is a homology functor.

We return in $\S 4$ to an explicit study of $h^{1}$ in special cases, obtaining the homology analog of Theorem 1.3. In this section we study a construction due to Adams(2) (unpublished) and prove a generalization of his theorem.

If $\mathbf{A}$ is an arbitrary abelian category, then a homology functor $h: \mathbf{T} \rightarrow \mathbf{A}$ from the triangulated category $\mathbf{T}$, with automorphism $\Sigma$, may be regarded as a functor $h: \mathbf{T} \rightarrow \mathbf{A}^{\mathbf{Z}}$, which commutes with $\Sigma$ (where $\Sigma: \mathbf{A}^{\mathbf{Z}} \rightarrow \mathbf{A}^{\mathbf{Z}}$ is the degree shift), and sends triangles to exact sequences. Let $\mathbf{T}\left[S^{-1}\right]$ be the category of fractions [7] with respect to $h$; that is, the category in which we render formally invertible the morphisms $f$ such that $h(f)$ is an isomorphism. These morphisms form the collection $S$. Further, let $F_{S}: \mathbf{T} \rightarrow \mathbf{T}\left[S^{-1}\right]$ be the canonical functor; since $F_{S}$ is the identity on objects, we will write $X$ for $F_{S}(X)$-and even sometimes $g$ for $F_{S}(g)$-if there is no danger of confusion. For each object $Y$ of $\mathbf{T}$, let $T_{Y}: \mathbf{T} \rightarrow \mathbf{A b}$ be the contravariant functor given by

$$
T_{Y}(X)=\mathbf{T}\left[S^{-1}\right](X, Y)
$$

Then the following is a generalization of Adams' theorem.

Theorem 3.2. In order that the object $Z$ in $\mathbf{T}$ represent the functor $T_{Y}$, that is, in order that there exist, for each $X$ in $\mathbf{T}$, an isomorphism

$$
\tau_{X}: \mathbf{T}\left[S^{-1}\right](X, Y) \cong \mathbf{T}(X, Z),
$$

functorial in $X$, it is necessary and sufficient that conditions (i) and (ii) below be satisfied; it is also necessary and sufficient that conditions (i) and (iii) be satisfied:

(i) There exists $e: Y \rightarrow Z$ in $S$.

(ii) Given $e^{\prime}: Y \rightarrow Z^{\prime}$ in $S$, there exists a unique $f: Z^{\prime} \rightarrow Z$ in $\mathbf{T}$ (and hence in S) such that $e=f e^{\prime}$.

(iii) If $h(X)=0$, then $\mathbf{T}(X, Z)=0$.

Proof. We prepare the way for the proof by making the following observations.

Proposition 3.3. $S$ admits a calculus of left fractions.

This is essentially Proposition 4.2 of [8].

(2) Communicated in letters of January 24 and February 20, 1969. 
Proposition 3.4. Given (i) and (ii), then each map $s: Z \rightarrow W$ in $S$ admits a retraction $r: W \rightarrow Z$ such that $r s=1$ (so that $r$ is also in $S$ ).

Proof of Proposition 3.4. By (ii) there exists $r: W \rightarrow Z$ such that $r s e=e$; and then, by the uniqueness of $f$ in (ii), $r s=1$.

Proof of Theorem 3.2. We first prove that, in the presence of (i), (ii) is equivalent to (iii). Assume (ii) holds and let $h(X)=0, g: X \rightarrow Z$. There is a triangle in $\mathbf{T}$,

$$
X \stackrel{g}{\longrightarrow} Z \stackrel{j}{\longrightarrow} W \stackrel{k}{\longrightarrow} \Sigma X,
$$

and, since $h$ is a homology functor, it follows that $h(j)$ is an isomorphism, so that $j$ is in $S$. By Proposition 3.4, there exists $r$ such that $r j=1$, so that $g=r j g=0$ and (iii) is proved.

Now suppose that (iii) holds. Let $e^{\prime}: Y \rightarrow Z^{\prime}$ be in $S$ and embed $e^{\prime}$ in the triangle

$$
Y \stackrel{e^{\prime}}{\longrightarrow} Z^{\prime} \stackrel{j}{\longrightarrow} W \stackrel{k}{\longrightarrow} \Sigma Y .
$$

Since $h$ is a homology functor and $h\left(e^{\prime}\right)$ is an isomorphism, $h(W)=0$, so that $\mathbf{T}\left(\Sigma^{i} W, Z\right)=0$, all $i$, by (iii). Thus

$$
\mathbf{T}\left(e^{\prime}, Z\right): \mathbf{T}\left(Z^{\prime}, Z\right) \cong \mathbf{T}(Y, Z)
$$

so that, in particular, there exists a unique $f: Z^{\prime} \rightarrow Z$ such that $f e^{\prime}=e$.

We next prove that (i), (ii) imply that there exists an isomorphism $\tau$ : $\mathbf{T}\left[S^{-1}\right](X, Y) \cong \mathbf{T}(X, Z)$, functorial in $X$. Now the transformation $\rho: \mathbf{T}(X, Z)$ $\rightarrow \mathbf{T}\left[S^{-1}\right](X, Z)$ is an isomorphism in view of Propositions 3.3, 3.4 above (see Proposition 4.1.2 of [7]). Also the transformation $\omega: \mathbf{T}\left[S^{-1}\right](X, Y) \rightarrow$ $\mathrm{T}\left[S^{-1}\right](X, Z)$ induced by $e: Y \rightarrow Z$ is certainly an isomorphism because $F_{S}(e)$ is invertible. Thus we may take $\tau=\rho^{-1} \omega$; then $\tau$ is obviously functorial in $X$ because $\rho$ and $\omega$ are functorial in $X$.

Finally we show that the existence of $\tau$ implies (i) and (ii). We define $e$ to be the image under $\tau$ of $1 \in \mathbf{T}\left[S^{-1}\right](Y, Y)$. To show $h(e)$ an isomorphism it of course suffices to show $F_{S}(e)$ an isomorphism. Let $\alpha \in \mathbf{T}\left[S^{-1}\right](Z, Y)$ be given by $\tau(\alpha)=1_{Z}$. We will show that $\alpha$ is inverse to $F_{S}(e)$. First the commutativity of

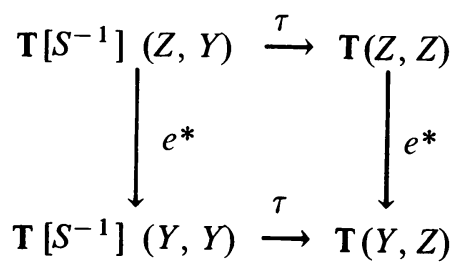

shows that $\tau\left(\alpha F_{S}(e)\right)=e=\tau(1)$, so that $\alpha F_{S}(e)=1$. Next, represent $\alpha$, as we may [7], by 


$$
Z \stackrel{f}{\longrightarrow} Y^{\prime} \stackrel{s}{\longleftarrow} Y,
$$

with $s$ in $S$, and consider the commutative diagram

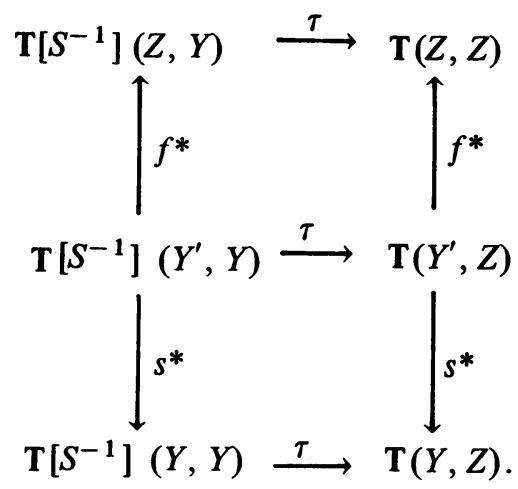

Let $\tau\left(F_{S}(s)^{-1}\right)=g$. Then $g f=\tau(\alpha)=1$, from the top square; and $g s=\tau(1)=e$, from the bottom square. Thus

$$
F_{S}(e) \alpha=F_{S}(g) F_{S}(s) F_{S}(s)^{-1} F_{S}(f)=F_{S}(g f)=1,
$$

so that $\alpha$ is inverse to $F_{S}(e)$.

Finally, we prove (ii). Given $e^{\prime}: Y \rightarrow Z^{\prime}$ in $S$, consider the commutative diagram

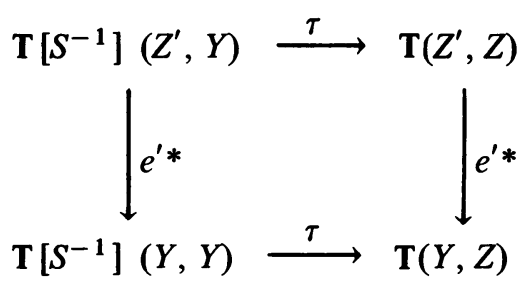

Then if $f=\tau\left(F_{S}\left(e^{\prime}\right)^{-1}\right), e=f e^{\prime}$; conversely if $e=f e^{\prime}$ and $f=\tau(\beta)$, then

$$
\tau\left(\beta F_{S}\left(e^{\prime}\right)\right)=e=\tau(1), \quad \beta F_{S}\left(e^{\prime}\right)=1,
$$

so

$$
\beta=F_{S}\left(e^{\prime}\right)^{-1} \text {. }
$$

Thus (ii) is established and hence Theorem 3.2 in its entirety.

We call $Z$ the (Adams) $h$-completion of $Y$ and write $Z=Y_{h}, e: Y \rightarrow Y_{h}$.

Remark. We have not made $\tau$ explicit, in this proof, in terms of $e$. Given $\alpha \in \mathrm{T}\left[S^{-1}\right](X, Y)$, represented by $X \stackrel{g}{\rightarrow} Y^{\prime} \stackrel{s}{\leftarrow} Y$, with $s$ in $S$, we find a unique $f: Y^{\prime} \rightarrow Z$ with $f s=e$ and then $\tau(\alpha)=f g$, for $e s^{-1} g=f g$ in $T\left[S^{-1}\right](X, Z)$, so that $\omega(\alpha)=\rho(f g)$. 
Applications. Our first application will be vital to the sequel. Let $\mathbf{Z}_{P}$ be the localization of the integers at the set of primes $P$ and let $\mathbf{J}$ be the category of based, 1-connected, CW-complexes and based maps. Then we may localize the objects of $\mathbf{J}$ [12]. Moreover, if $\tilde{H}_{*}$ stands for (reduced) ordinary homology,

$$
\tilde{H}_{*}\left(X_{P}\right)=\tilde{H}_{*}(X) \otimes \mathbf{Z}_{P},
$$

where $X_{P}$ is the localization of $X$ at $P, X$ in $\mathbf{J}$. Since $\mathbf{Z}_{P} \otimes \mathbf{Z}_{P} \cong \mathbf{Z}_{P}$ and $\mathbf{Z}_{P}$ is flat [12], we may make (3.2) more precise by remarking that the localization map $l: X \rightarrow X_{P}$ (which is defined up to homotopy [12]) induces an isomorphism $l_{*}: \tilde{H}_{*}\left(X ; \mathbf{Z}_{P}\right) \cong \tilde{H}_{*}\left(X_{P} ; \mathbf{Z}_{P}\right)$. Moreover, by Theorem $1.1, l$ has the universal property expressed by the fact that if $f: X \rightarrow X^{\prime}$ induces an isomorphism in homology with $\mathbf{Z}_{P}$ coefficients, then there exists $u: X^{\prime} \rightarrow X_{P}$, unique up to homotopy, such that $u f \simeq l$.

Let us take $\mathbf{T}$ to be the stabilization of $\mathbf{J}$; obviously, localization commutes with stabilization. Of course, the restriction that the objects of $\mathbf{J}$ be 1 -connected has no effect in $\mathbf{T}$. Let $h: \mathbf{T} \rightarrow \mathbf{A b}$ be the homology functor which comes from stabilizing $\tilde{H}_{*}\left(-; \mathbf{Z}_{P}\right)$. Then the facts claimed above, together with Theorem 3.2, show that the functor $T_{(Y, m)}$ of (3.1) is represented by $\left(Y_{P}, m\right)$. Indeed, conditions (i), (ii) of Theorem 3.2 hold where the role of $e$ is played by the stabilization of the homotopy class of $l$, and $Z=\left(Y_{P}, m\right)$. We will see in the next section (Lemma 4.2) that $\left(Y_{P}, m\right)=(Y, m) \wedge K^{\prime}\left(\mathbf{Z}_{P}, 0\right)$, where $K^{\prime}$ stands for the stable Moore space.

As a second application we may let $h: \mathbf{T} \rightarrow \mathbf{A b}$ be the homology functor which comes from stabilizing $\tilde{H}_{*}(-; \mathbf{Z} / p \mathbf{Z})$. In this case, as already remarked by Adams, the functor $\mathbf{T}_{(Y, m)}$ of $(3.1)$ is represented by $\left(\hat{Y}_{P}, m\right)$, where $\hat{Y}_{P}$ is the $p$ profinite completion of $Y$ in the sense of [12]. Thus the Adams completion generalizes both localization and $p$-profinite completion in the stable category.

As a third application, let us take $\mathbf{T}$ to be the Boardman category of spectra $\mathbf{B}$ [13]. Then by Proposition 4.2 and 3.2 of [8], for any homology functor $h: \mathbf{B} \rightarrow \mathbf{A} \mathbf{b}^{\mathbf{Z}}$, the category of fractions $\mathbf{B}\left[S^{-1}\right]$ has a unique structure of triangulated category such that the canonical functor $F_{S}: \mathbf{B} \rightarrow \mathrm{B}\left[S^{-1}\right]$ commutes with the automorphism $\Sigma$ and takes triangles into triangles. Moreover, $F_{S}$ takes coproducts in $\mathbf{B}$ into coproducts in $\mathbf{B}\left[S^{-1}\right]$. Thus for any spectrum $Y \in|\mathbf{B}|$ the functor $T_{Y}: \mathbf{B} \rightarrow \mathbf{A b}$ of (3.1) is a generalized cohomology theory [13, p. 178], and it is known that any such theory is representable [13, p. 180]. Hence there exists a spectrum $Z \in|\mathbf{B}|$ such that $\mathbf{B}\left[S^{-1}\right](X, Y)=T_{Y}(X) \cong \mathbf{B}(X, Z)$ functorially in $X$. Thus in the Boardman category the Adams completion always exists.

As a fourth, and rather different, application, let $\mathbf{A}$ be an arbitrary abelian category and let $\mathbf{T}$ be the category $\mathbf{K}$ of cochain-complexes in $\mathbf{A}$ and homotopy classes of cochain-maps. Then $h: \mathbf{T} \rightarrow \mathbf{A}$ is the homology functor and $\mathbf{T}\left[S^{-1}\right]$ is the derived category [8] of $\mathbf{A}$, which we will write as $\mathbf{D}$. Assume that $\mathbf{A}$ has enough injectives and that $Y$ is an object of $\mathbf{K}$ which is bounded below (i.e., $Y_{n}=0$ for 
$n<n_{0}$ ). Then (Lemma 4.6 of [8]), there exists $e: Y \rightarrow I$ in $\mathbf{K}$ such that $I$ is bounded below and consists of injective objects and $h(e)$ is an isomorphism. In fact, it then follows that $T_{Y}$ (see (3.1)) is represented by $I$, so that $D(X, Y)$ $\cong \mathbf{K}(X, I)$ functorially in $X$. To see this we only have to verify that $\mathbf{K}(X, I)=0$ if $h(X)=0$, but this is ensured by Lemma 4.4 of [8].

Remark. The following alternative approach might be used to study the Adams completion.

Freyd has constructed for any triangulated category $\mathbf{T}$ a canonical imbedding $I: \mathbf{T} \rightarrow \Phi \mathbf{T}$ into a Frobenius abelian category $\Phi \mathbf{T}$. (See for instance [9].) Then for any homology functor $h: \mathbf{T} \rightarrow \mathbf{A}^{\mathbf{Z}}$ there exists a unique exact functor $\Phi h: \Phi \mathbf{T} \rightarrow \mathrm{A}^{\mathrm{Z}}$ such that $h=\Phi h \circ I$. Let $R$ be the collection of all morphisms $\phi$ in $\Phi$ T such that $\Phi h(\phi)$ is an isomorphism. Then we have a commutative diagram of categories and functors

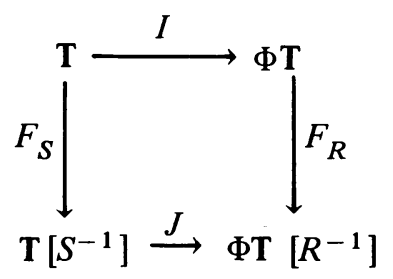

The full subcategory $\operatorname{ker}(\Phi h)$ of $\Phi \mathrm{T}$ consisting of those objects sent to zero by $\Phi h$ is then a "thick" (épaisse) subcategory of $\Phi T$ and the category of fractions $\Phi \mathbf{T}\left[R^{-1}\right]$ is identified with the quotient category $\Phi \mathbf{T} / \operatorname{ker}(\Phi h)$ (see p. 15 of [7]). One could then apply the techniques of Gabriel's thesis [6].

4. Serre classes and Kan extensions. Let $T$ be a triangulated category and let $h, k$ be two homology theories on $\mathbf{T}$. Let $X$ in $\mathbf{T}$ admit the Adams $h$-completion $X_{h}$, and form the triangle

$$
X \stackrel{e}{\longrightarrow} X_{h} \stackrel{i}{\longrightarrow} C_{e} \stackrel{j}{\longrightarrow} \Sigma X
$$

in $\mathbf{T}$.

Let $\mathbf{T}_{0}$ be the full subcategory of $\mathbf{T}$ whose objects are those $Y$ such that $h(Y)=0$. It is plain that $\mathbf{T}_{0}$ is a triangulated subcategory of $\mathbf{T}$. Let $k^{0}$ be the restriction of $k$ to $\mathbf{T}_{0}$, and let $k^{1}$ be the Kan extension of $k^{0}$ to $\mathbf{T}$. We prove

Theorem 4.1. $k_{n}^{1}(X)=k_{n+1}\left(C_{e}\right)$.

Proof. Since $h(e)$ is an isomorphism, it is clear from (4.1) that $h\left(C_{e}\right)=0$, so that $C_{e}$ is in $\mathbf{T}_{0}$. We will show that, in the category of $\mathbf{T}_{0}$-objects over $X$, the singleton $\Sigma^{-1} j: \Sigma^{-1} C_{e} \rightarrow X$ is cofinal. This will establish the theorem, since it then follows from Theorem 1.4 that $k_{n}^{1}(X)=k_{n}\left(\Sigma^{-1} C_{e}\right)=k_{n+1}\left(C_{e}\right)$. Thus, suppose $h(Y)=0$ and consider $f: Y \rightarrow X$ in T. By (iii) of Theorem 3.2, ef $=0$, so $f$ factors as $\left(\Sigma^{-1} j\right) g, g: Y \rightarrow \Sigma^{-1} C_{e}$. However since $\mathbf{T}\left(Y, \Sigma^{-1} X_{h}\right)=0$, it follows that the 
factorization $f=\left(\Sigma^{-1} j\right) g$ determines $g$ uniquely, so that the singleton $\Sigma^{-1} j$ is indeed cofinal as claimed.

We apply this theorem to the case where $\mathbf{T}$ is the stable $\mathbf{C W}$-category $\mathbf{S}$, that is, the stabilization of the category of $\mathrm{CW}$-complexes, and $h=\tilde{H}_{*}\left(-; \mathbf{Z}_{\bar{P}}\right)$, where $P$ is a family of primes and $\bar{P}$ is the complementary family. We first prove two lemmas.

Lemma 4.2. If $Q$ is a family of primes and if $h=\tilde{H}_{*}\left(-; \mathbf{Z}_{Q}\right)$, then $X_{h}$ $=X \wedge K^{\prime}\left(\mathbf{Z}_{Q}, 0\right)$, where $K^{\prime}$ stands for the stable Moore space.

Proof. The map $e: X \rightarrow X \wedge K^{\prime}\left(\mathbf{Z}_{Q}, 0\right)$ is just obtained from the embedding $\mathbf{Z} \subseteq \mathbf{Z}_{Q}$, where $X$ is identified with $X \wedge K^{\prime}(\mathbf{Z}, 0)$. It is then plain that $\tilde{H}_{*}(e)$ : $\tilde{H}_{*}(X) \rightarrow H_{*}(X) \otimes \mathbf{Z}_{Q}$ simply localizes $\tilde{H}_{*}(X)$. Thus $X \wedge K^{\prime}\left(\mathbf{Z}_{Q}, 0\right)$ is the $Q$ localization of $X$, so that, according to our first application of Theorem 3.2, it is also the $h$-completion $X_{h}$ of $X$.

Lemma 4.3. Let $C_{P}$ be the Serre class of $P$-torsion groups. Then $A \in C_{P}$ iff $A \otimes \mathbf{Z}_{\bar{P}}=0$.

Proof. Write $n \in P$ if $n$ is a product of primes in $P$. If $a \in A \in C_{P}$ then $n a=0$ for some $n \in P$, so that, if $q \in \mathbf{Z}_{\bar{P}}, a \otimes q=n a \otimes q / n=0$. Conversely, suppose $A \otimes \mathbf{Z}_{\bar{P}}=0$. Then $A$ is plainly a torsion group, since $\mathbf{Z}_{\bar{P}}$ is torsion free. Moreover, since $\mathbf{Z} / p^{n} \mathbf{Z} \otimes \mathbf{Z}_{\bar{P}} \cong \mathbf{Z} / p^{n} \mathbf{Z}$ if $p \in \bar{P}$ [12], it follows that $A$ has no $\bar{P}$ torsion, so $A \in C_{P}$.

From these lemmas we deduce the following theorem; as in [5], we write $Y \in C_{P}$ to indicate that the homology and homotopy groups of the (stable) space $Y$ belong to $C_{P}$.

Theorem 4.4. Let $\mathbf{S}$ be the stable $C W$-category and let $\mathbf{S}_{0}$ be the full subcategory consisting of spaces in $C_{P}$. If $k$ is a homology theory on $\mathbf{S}$ and if $k^{1}$ is the Kan extension to $\mathbf{S}$ of the restriction of $k$ to $\mathbf{S}_{0}$, then, for $X$ in $\mathbf{S}$,

$$
k_{n}^{1}(X)=k_{n+1}\left(X ; \mathbf{Z}_{P^{\infty}}\right) \text {. }
$$

Here $\mathbf{Z}_{P^{\infty}}$ is, of course, the (generalized) Prüfer group consisting of the direct sum of the $p$-primary components of $\mathbf{Q} / \mathbf{Z}$ for all $p \in P$.

Proof. By Lemma $4.3, \mathrm{~S}_{0}$ is the subcategory given by $h(Y)=0$ where $h=\tilde{H}_{*}\left(-; \mathbf{Z}_{\bar{P}}\right)$. By Theorem 4.1, $k_{n}^{1}(X)=k_{n+1}\left(C_{e}\right)$, where $X \stackrel{e}{\rightarrow} X_{h} \stackrel{i}{\rightarrow} C_{e}$ $\stackrel{j}{\rightarrow} \sum X$ is a triangle in $\mathbf{S}$ and $X_{h}$ is the Adams $h$-completion of $X$. By Lemma 4.2, $X_{h}=X \wedge K^{\prime}\left(\mathbf{Z}_{\bar{P}}, 0\right)$, so that

$$
C_{e}=X \wedge K^{\prime}\left(\mathbf{Z}_{\bar{P}} / \mathbf{Z}, 0\right) .
$$

But $\mathbf{Z}_{\bar{P}} / \mathbf{Z}=\mathbf{Z}_{P^{\infty}}$; and it only remains to note that, by definition, one puts coefficients $G$ into the homology theory $k$ by the rule [10] $k_{r}(X ; G)=$ $k_{r}\left(X \wedge K^{\prime}(G, 0)\right)$.

Remarks. (i) Theorem 4.4 is, of course, the homology analog of Theorem 1.3. By working stably, and by replacing cohomology by homology, considerable 
simplification has been achieved. On the other hand, Theorem 2.2 shows that we were free to work stably in our proof of Theorem 1.3 in [5]. The proof of Theorem 4.4 is, however, indirect, so that it is not clear how we would generalize to arbitrary Serre classes.

(ii) Lemma 4.3 implies that $Y \in C_{P}$ iff $\tilde{H}_{*}\left(Y ; \mathbf{Z}_{\bar{P}}\right)=0$. Evidently an alternative criterion is that $Y_{h} \simeq 0$ where $h=\tilde{H}_{*}\left(-; Z_{\bar{P}}\right)$. Now we may localize the category $\mathbf{S}$ at $\bar{P}$ defining $\mathbf{S}_{\bar{P}}(A, B)=\mathbf{S}(A, B) \otimes Z_{\bar{P}}$. It is then plain that if $Y \simeq 0$ in $\mathbf{S}_{\bar{P}}$ (we may write this: $Y \simeq_{\bar{P}} 0$ ), then $Y \in C_{P}$. For if $Y \simeq_{\bar{P}} 0$, then $n 1=0: Y \rightarrow Y$ for some $n \in P$, so that $1=0: \tilde{H}_{*}(Y) \otimes Z_{\bar{P}} \rightarrow \tilde{H}_{*}(Y) \otimes Z_{\bar{P}}$. An easy inductive argument shows that, conversely, $Y \simeq_{\bar{P}} 0$ if $Y \in \mathcal{C}_{P}$, provided that $Y$ is finitedimensional. In fact, localizing the category $\mathbf{S}$ at $\bar{P}$ is equivalent to constructing the category of fractions of $\mathbf{S}$ in which one formally inverts the morphisms in $\mathbf{S}$ of the form $m l_{X}$, where all the primes which divide the integer $m$ belong to $P$, and $1_{X}$ is the identity morphism of the object $X$ of $\mathbf{S}$.

\section{REFERENCES}

1. M. Artin and B. Mazur, Etale homotopy, Lecture Notes in Math., no. 100, Springer-Verlag, Berlin and New York, 1969. MR 39 \#6883.

2. A. Bousfield and D. Kan, Homotopy with respect to a ring, Proc. Sympos. Pure Math., vol. 22, Amer. Math. Soc., Providence, R. I., 1971, pp. 59-64.

3. A. Deleanu and P. Hilton, On the general Čech construction of cohomology theories, Symposia Mathematica Ist. Naz. di Alt. Mat., 1970, pp. 193-218.

4.--, Remark on Čech extension of cohomology functors, Proc. Adv. Stu. Inst. Alg. Top., Aarhus, 1970 pp. 44-66.

5.—, On Kan extensions of cohomology theories and Serre classes of groups, Fund. Math. 73 (1971), 143-165; see also: Battelle Institute Report No. 34, 1970.

6. P. Gabriel, Des catégories abéliennes, Bull. Soc. Math. France 90 (1962), 323-448. MR 38 \# 1144.

7. P. Gabriel and M. Zisman, Calculus of fractions and homotopy theory, Ergebnisse der Mathematik und ihrer Grenzgebiete, Band 35, Springer-Verlag, New York, 1967. MR 35 \# 1019.

8. R. Hartshorne, Residues and duality, Lecture Notes in Math., no. 20, Springer-Verlag, Berlin and New York, 1966. MR 36 \#5145.

9. A. Heller, Stable homotopy categories, Bull. Amer. Math. Soc. 74 (1968), 28-63. MR 36 \#137.

10. P. Hilton, Putting coefficients into a cohomology theory. I,II, Nederl. Akad. Wetensch. Proc. Ser. A 73 = Indag. Math. 32 (1970), 196-209, 210-216. MR 42 \#1105a, b.

11. J. Milnor, On spaces having the homotopy type of a $\mathrm{CW}$-complex, Trans. Amer. Math. Soc. 90 (1959), 272-280. MR 20 \#6700.

12. D. Sullivan, Geometric topology. I. Localization, periodicity and Galois symmetry, M.I.T., Cambridge, Mass., 1970 (mimeographed notes).

13. R. Vogt, Boardman's stable homotopy category, Lecture Notes Series, no. 21, Matematisk Institut, Aarhus Universitet, Aarhus, 1970. MR 43 \# 1187.

Department of Mathematics, Syracuse University, Syracuse, New York 13210 (Current Address of Aristide Deleanu)

Battelle Seattle Research Center, Seattle, Washington 98105 (Current Address of Peter Hilton)

Department of Mathematics, University of Washington, Seattle, Washington 98195 